\title{
Can the observed vibration of a cantilever of supersmall mass be explained by quantum theory?
}

\author{
Alekber Yu. Kasumov, Nikolai A. Kislov and Igor I. Khodos \\ Institute of Microelectronics Technology and High Purity Materials, Russian Academy of Sciences, \\ 142432, Chernogolovka, Moscow Region, Russia
}

(Received October 09, 1992; accepted September 27, 1993)

\begin{abstract}
An attempt has been made to test experimentally the consequences of the "alternative quantum theory" by electron microscopy technique. It has been found that the free end of a cantilever of $10^{-15}-10^{-16} \mathrm{~g}$ in mass performs chaotic jumps of $\lesssim 100 \AA$ amplitude; the mean frequency of jumps of $\approx 20-30 \AA$ amplitude is $\approx 1 \mathrm{~Hz}$. An increase in the mass of the object under observation up to $(5-7) \cdot 10^{-15} \mathrm{~g}$ by growing the load on the free end of cantilever results in damping the vibrations for 5 - 10 days. It has been deduced that the obtained results confirm the macroscopic center-of-mass quantum fluctuations and spontaneous localization of the object, predicted by the theory.
\end{abstract}

\section{Introduction.}

Traditional quantum mechanics has not introduced any criterion for separating microscopic quantum and macroscopic bodies [1]. The motion of a body of any mass can be described by the wave function and its spreading with time should give rise to "monstrous" quantum states of macroscopic bodies [2]. It would be natural to ask why no such states have ever been observed in $\mathrm{Na}$ ture, and this question is basic in quantum mechanics, since it is tightly related to the problems of measurements and the Observer.

The generally accepted viewpoint consists in a statement that the body "is being continuously measured" by its environment. As a result, the wave packet connected with the body is reduced and body's behaviour is classical [3]. Such an approach leads to different paradoxes (for instance, "the Schrödinger cat" [4]), their resolution in the framework of the traditional quantum mechanics is rather difficult.

In an approach alternative to the generally accepted theory of the Copenhagen school, the reduction of a wave packet has been accounted for by the self gravitational effect of the body, preventing the wave packet from spreading [5]. In this approximation the motion of a body is described by the modified nonlinear Schrödinger equation, into which an additional term, arising from the body's self-gravitation, is introduced. Proceeding from this concept, all bodies (from an elementary particle to astronomical objects) possess quantum properties, but the quantum state of any physical body is metastable: after some time, $\tau$, macroscopic quantum fluctuations (of the 
center-of-mass, for instance) are damping. In a quantum state the body should perform Brownian movement, damping with time, which is not caused by any external classical forces [6-8]. The time, $\tau$, when a body is in a quantum state depends on its mass. The lifetime of such a quantum state can be given by the well-known equation [1]:

$$
\tau=\hbar / \Gamma
$$

Here $\Gamma=G M^{2} / R$ is the energy level width, $G$ is the gravitational constant, $M$ is the mass of the body, $R$ is the size of the body. For elementary particles and atoms the gravitation term is small, and, hence, $\tau>10^{16} \mathrm{~s}$, i.e., the time is longer than the age of the Universe. For macroscopic bodies the time $\tau$ is very short. For the body of $10^{-15}-10^{-16} \mathrm{~g}$ in mass and $10^{-5} \mathrm{~cm}$ in size the quantum state's lifetime amounts to $10^{5}-10^{7} \mathrm{~s}$, that allows observing such a state in experiment.

But as far as we know, in none of experiments this concept [5] has been verified up to now. The main difficulty in the observation of "new" quantum properties is the production of rather free bodies of an extremely small mass (which must be much smaller than that of common macroscopic bodies and, at the same time, much greater than the atoms), and the observation of their position in space with the high precision. Provided the mass of a body amounts to $10^{-15}-10^{-16} \mathrm{~g}$ and the rate of motion of the order of $1 \AA / \mathrm{s}$, the length of the de Broglie wave associated with it is equal to $100-1000 \mathrm{~nm}$, which much exceeds the side of the body itself. And it is thus possible to observe quantum features in the behaviour of such bodies.

But the observation of quantum behaviour of suspended dust particles, discussed in some theoretical papers (see, for example, [9]), cannot in our opinion be realized in experiment. Small particles, deposited on the substrate, cannot be regarded as suitable objects because of their strong interaction with such a more massive substrate, and they practically form a single body with it. In a number of papers devoted to the problem of quasimelting of small gold particles [10-12], it was possible to diminish this interaction. The particle of $<10 \mathrm{~nm}$ in size displayed chaotic motion, but the reason of such a motion has not been elucidated [12]. In experiments, aimed at studying fringes in an electron-microscopic pattern of protein fibrils, permanent motion of a small fibril of $\sim 6 \mathrm{~nm}$ in diameter and $\sim 100 \mathrm{~nm}$ in length, positioned on a carbon film with holes, was observed. However, no reasons of the motion of a fibril were discussed in the paper.

It should be noted that in the above papers the study of the dynamics of these bodies was not the purpose of the experiments. Yet, in our opinion, the study of the dynamics of nanoobjects is of fundamental interest. We believe that the use of an electron microscope may assist in creating small bodies of the required mass and their quantum features can be observed in their dynamics. Proper objects may be self-supporting carbon rods grown outside the substrate in the scanning mode of an electron microscope [14]. These rods can be considered as quasi-free when studying their quantum properties.

\section{Experiment.}

We have produced a body of a required small mass in a scanning transmision electron microscope, VG HB501, with a $10 \AA$ beam diameter, using carbon deposition stimulated by an electron beam. While the electron beam was moving from substrate's edge, a self-supporting rod (cantilever) was grown with one end fastened to the edge of the substrate [15, 16] (Fig. 1). It consists of amorphous hydrocarbons $[14,16]$. Typical sizes of rods are $200-500 \AA$ in width and $0.2-2.0 \mu \mathrm{m}$ in length. The displacements of the grown cantilever free end relative to the fixed reference point were observed on the screen of a transmission electron microscope, JEM-2000FX, with $10^{6}-10^{7}$ magnification, involving a $\times 10$-fold magnification caused by the binocular. We were able to detect 
minimal displacements amounting to $\leq 5 \AA$. The image drift was $\leq 0.2 \AA / \mathrm{s}$. Observations were performed at a very low density of the beam current (about $0,1 \mathrm{pA} / \mathrm{cm}^{2}$ ) in order to exclude buildup of new carbon layers on the cantilever and reduce the electrostatic forces.

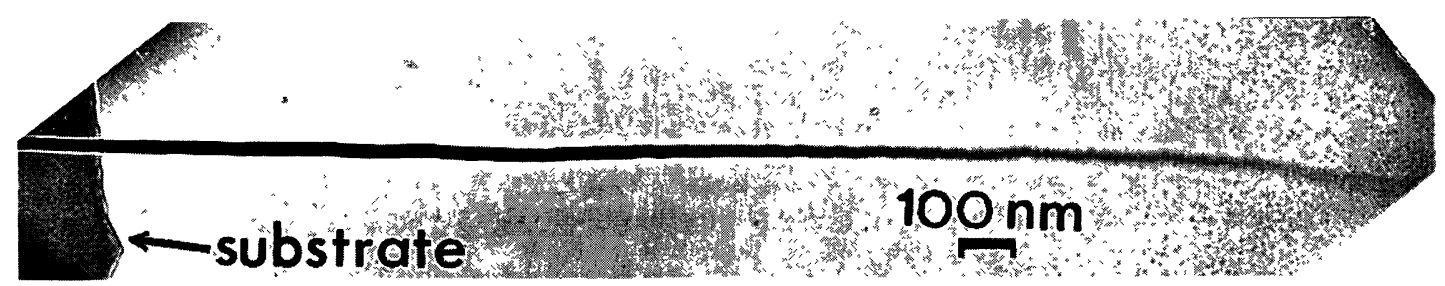

Fig. 1. - General view of a cantilever (TEM micrograph).

\section{Results and discussion.}

Cantilever free ends performed random jumps. The length of jumps changed chaotically and reached the maximum value (100-150 $\AA$ ) with the frequency of $0.1-0.2 \mathrm{~Hz}$ (Tab. I). The mean frequency of jumps of $\approx 10-30 \AA$ in length equaled $\approx 1 \mathrm{~Hz}$. Sometimes two or more subsequent jumps occurred in the same direction. The blurring of the cantilever image in figure 1 could be due to this vibration (the cantilever image becomes more and more diffuse towards its free end; the exposure time was $8 \mathrm{~s}$ ). At the same time the images of the substrate and of the basis of the cantilever are sharp in this figure.

Let us consider the possible reasons which might induce vibrations of cantilever.

1) Mechanical vibrations of the cantilever support. None of them were observed in our experiments (as one may see from figure 1 the rod bottom and substrate are depicted sharp). Besides, the resonant frequency of the cantilever vibrations has to be $10^{6}-10^{8} \mathrm{~s}^{-1}$, so the observed vibrations (with frequency $0.1-1 \mathrm{~s}^{-1}$ ) cannot be related to the resonant effect.

2) Time-variable forces, induced by heating the cantilever with an electron beam and also by electrostatic or electrodynamic effects of the beam. The effect of these forces is negligible because a change of the beam current by a factor of 10 (from $0.1 \mathrm{pA} / \mathrm{cm}^{2}$ to $1 \mathrm{pA} / \mathrm{cm}^{2}$ ) did not affect either the amplitude or the vibration frequency. Besides, to eliminate such an effect of the beam, we have grown cantilevers on metallic substrates and then coated them with a thin layer of metal; and in this case vibrations of the cantilever did not vanish either.

3) Vibrations set up by impacts of residual gas molecules against the cantilever. The observations show that the amplitude and frequency of vibrations do not depend on the vacuum level in the microscope column which could be modified by a liquid nitrogen cooled trap. Note that the theoretically estimated value of virtual deflection of cantilevers resulting from molecule impacts is below the detection limit.

It should be emphasized that the classical external forces mentioned above cause cantilevers of lower rigidity to be displaced to a greater extent [17]. In our case the average amplitude of the displacement depends on the cantilever mass as well, therefore the displacement of lower rigidity cantilevers can be smaller. For instance, the rigidity coefficient of the cantilevers No 13-15 (Tab I) is substantially lower than that of cantilevers No 7-12, while the vibration amplitude of the latter 
Table I. - Cantilever parameters and observed mean amplitudes of vibrations.

\begin{tabular}{|l|c|c|c|c|c|}
\hline $\begin{array}{c}\text { Sample } \\
\text { number }\end{array}$ & $\begin{array}{c}\text { Length } l, \\
10^{-4} \mathrm{~cm}\end{array}$ & $\begin{array}{c}\text { Width } d, \\
\AA\end{array}$ & $\begin{array}{c}\text { Mass } M^{*}, \\
\times 10^{-16} \mathrm{~g}\end{array}$ & $\begin{array}{c}\text { Rigidity } K^{* *}, \\
\text { dyn } / \mathrm{cm}\end{array}$ & $\begin{array}{c}\text { Mean } \\
\text { amplitude } \\
\Delta x, \AA\end{array}$ \\
\hline 1 & 2.0 & 200 & 32 & 0.1 & 150 \\
$2^{* * *}$ & 1.9 & 250 & 91 & 0.28 & 30 \\
$3^{* * *}$ & 1.1 & 200 & 60 & 0.6 & 30 \\
$4^{* * * *}$ & 0.51 & 200 & 9.6 & 6 & 30 \\
5 & 0.40 & 200 & 6.5 & 12.5 & 20 \\
6 & 0.42 & 200 & 6.9 & 11.8 & 20 \\
7 & 0.20 & 150 & 1.8 & 320 & 20 \\
8 & 0.19 & 150 & 2.0 & 360 & 20 \\
9 & 0.34 & 250 & 8.3 & 57 & 10 \\
10 & 0.39 & 350 & 19.2 & 126 & 10 \\
11 & 0.31 & 350 & 15.2 & 260 & 5 \\
12 & 0.18 & 250 & 4.5 & 340 & 5 \\
13 & 1.8 & 500 & 181 & 4.7 & 0 \\
14 & 1.95 & 550 & 236 & 6.2 & 0 \\
15 & 1.6 & 600 & 232 & 15.9 & 0 \\
\hline
\end{tabular}

${ }^{*}$ mass was estimated from the mean density of the carbon fibres $\left(\approx 2 \mathrm{~g} / \mathrm{cm}^{3}[18]\right)$;

${ }^{* *}$ rigidity $\kappa$ was estimated taking $E=10^{11} \mathrm{dyn} / \mathrm{cm}^{2}$ (bend rigidity of the carbon fibres [18]) and $J=d^{3} b / 12$ - the rotary inertia of the rectangular rod ( $b$ is thickness of the rod); in our case $b \approx 2 \mathrm{~d}$

*** cantilevers with the load on the free end; the time of vibration damping for cantilever No 2 is 4 days, for cantilever No 3 is 11 days;

**** cantilever on the metal substrate.

is bigger. A progressive increase of loading on cantilevers No 2 and 3 resulted in a vibration amplitude some times smaller (e.g., with cantilever No 2 it was 5 times smaller).

The observed vibrations of cantilevers cannot thus be caused by any classical external forces. In our viewpoint it could be assumed that the jumps of cantilever are caused by quantum potential [19]. The kinetic energy, $T$, required for the cantilever of rigidity $K$ to deflect, as a result of an external impact (strike, impulse), by the value of $\Delta x$, can be found from the equation of the strength of materials theory [17]:

$$
T=\frac{K}{2}(\Delta x)^{2}
$$

where $K=3 E J / l^{3}, E$ is the bend rigidity, $J$ is the rotary inertia of the rod, $l$ is the length of the rod. For $\Delta x \approx 100 \AA, T \approx 10^{-12} \mathrm{erg}$. It follows directly from the uncertainty principle that a body of mass $M$ has a kinetic energy $\approx \hbar^{2} / 2 M(\Delta q)^{2}$. Provided its center-of-mass is localized in the region of $\Delta q \approx 10^{-13} \mathrm{~cm}$ (the size of an atomic nucleus) this energy will equal the above value of the kinetic energy $\left(10^{-12} \mathrm{erg}\right)$. The localization of a solid body to such an accuracy is possible [20].

To verify the deduction of the theory $[6,7]$ on the Brownian movement damping with growing 
mass we increased the mass of a cantilever without changing its rigidity. For this purpose a sphereshaped load of $\approx 10^{-5} \mathrm{~cm}$ in diameter was grown on a free end of the cantilever, a stationary electron beam being applied (Fig. 2). It is a most important experimental fact that the vibrations of cantilevers with a load on the end damped out over 5-10 days before an unobserved value was achieved (varying with the mass of consoles (Tab. I)). This time of vibration damping fits that predicted by theory, $\tau=10^{5} \mathrm{~s}$, for bodies of $10^{-5} \mathrm{~cm}$ in size [6,7]. At the same time cantilevers without loads vibrate with the same amplitude and frequency, as in the previous case. According to the estimation performed above, for these cantilevers $\tau=10^{7} \mathrm{~s}$, i.e. it is about a year. Whereas the time of fluctuation damping for microobjects (elementary particles, atoms, molecules) exceeds the age of the Universe, for these objects the predictions of the traditional quantum mechanics are valid $[6-8]$.

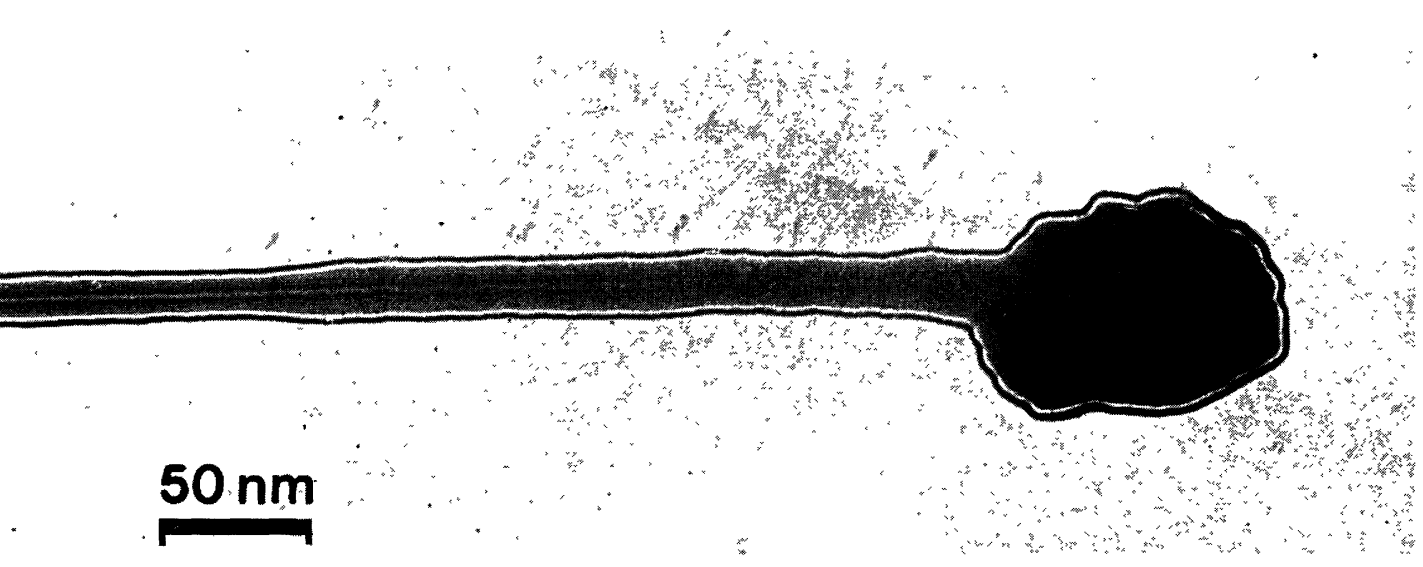

Fig. 2. - TEM micrograph of a cantilever with a load grown on the free end.

\section{Conclusions.}

Both the chaotic vibrations of the cantilever ends and their attenuation with time observed in the TEM could only be explained as macroscopic quantum fluctuations of the cantilever mass center. Thus, this paper reports for the first time experimental observations supporting the theory $[6,7]$ according to which the reduction of a wave packet is caused by gravitation forces, and not by the event of measurement and presence of an Observer.

\section{References}

[1] BoHM D., Quantum theory (Prentice-Hall, New York, 1952).

[2] SHImONy A., Philosophical Consequences of Quantum Theory, J. Cushing and E. Mc Mullin Eds. (Notre Dame Press, Notre Dame, 1990).

[3] ZEH H.D., Found. Phys. 1 (1970) 69. 
[4] SCHRÖDINGER E., Naturwiss. 23 (1935) 807.

[5] Penrose R., Quantum Concepts in Space and Time, R. Penrose and C.J. Ishani Eds. (Clarendon, Oxford, 1986) 129.

[6] Diosi L., Phys. Rev. A40 (1989) 1165.

[7] GHIRARDI G.C., Grassi R. and RIMINI A., Phys. Rev. A42 (1990) 1057.

[8] Ghirardi G.C., Pearle P. and Rimini A., Phys. Rev. A42 (1990) 78.

[9] JOOS E. and ZEH H.D., Z. Phys. B59 (1985) 223.

[10] BOVIN J., WALLENBERG R. and SMITH D., Nature 47 (1985) 317.

[11] IIJIMA S., ICHIHASHI T., Phys. Rev. Lett. 56 (1986) 616.

[12] AJAYAN P., MARKs L., Phys. Rev. Lett. 63 (1989) 279.

[13] Mollenstedt G. and PeTERS H.M., Proc. 2nd Inst. Symp. Foundations of Quantum Mechanics (Tokyo, 1986) 106.

[14] KIsLOV N.A. and KHOdos I.I., Microsc. Microanal. Microstr. 3 (1992) 323.

[15] PeASE R.F.W. and NixON W.C., Proc. 1st Internat. Conf. Electron Ion Beam Sci. Tech. (1965) 220.

[16] ARISTOV V.V., KISLOV N.A., KHODOS I.I., Microsc. Microanal. Microstr. 3 (1992) 313.

[17] TIMOSHENKo S., Strength of Materials (D. Van Nostrand Company. Inc., Princeton, New Jersey, 1955).

[18] FITZER E., Carbon Fibres and Their Composits (Springer-Verlag, 1985).

[19] BOHM D., Phys. Rev. 85 (1952) 166, 180

[20] CAVEs C.M., ThORNe K.S., DReVER R.W.P., SANDberg V.D. and Zimmerman M., Rev. Mod. Phys. 52 (1980) 341. 